\title{
Hospital Closure and Insights into Patient Dispersion
}

\section{The Closure of Saint Vincent's Catholic Medical Center in New York City}

N. Garg1; G. Husk2; T. Nguyen²; A. Onyile1; S. Echezona²; G. Kuperman³, 4. J.S. Shapiro

${ }^{1}$ Icahn School of Medicine at Mount Sinai, Emergency Medicine, New York, New York, United States;

2 Mount Sinai - Beth Israel, Emergency Medicine, New York, New York, United States;

${ }^{3}$ Department of Information Systems, New York-Presbyterian Hospital, New York, New York, United States;

${ }^{4}$ Department of Biomedical Informatics, Columbia University, New York, New York, United States

\section{Keywords}

Health information systems, health services research, health facility closure, medical informatics, health policy

\section{Summary}

Background: Hospital closures are becoming increasingly common in the United States. Patients who received care at the closing hospitals must travel to different, often farther hospitals for care, and nearby remaining hospitals may have difficulty coping with a sudden influx of patients. Objectives: Our objectives are to analyze the dispersion patterns of patients from a closing hospital and to correlate that with distance from the closing hospital for three specific visit types: emergency, inpatient, and ambulatory.

Methods: In this study, we used data from a health information exchange to track patients from Saint Vincent's Medical Center, a hospital in New York City that closed in 2010, to determine where they received emergency, inpatient, and ambulatory care following the closure.

Results: We found that patients went to the next nearest hospital for their emergency and inpatient care, but ambulatory encounters did not correlate with distance.

Discussion: It is likely that patients followed their ambulatory providers as they transitioned to another hospital system. Additional work should be done to determine predictors of impact on nearby hospitals when another hospital in the community closes in order to better prepare for patient dispersion.

\section{Correspondence to:}

Jason S. Shapiro

Department of Emergency Medicine,

Icahn School of Medicine at Mount Sinai, Box 1620,

One Gustave Levy Place, New York, NY 10029, USA;

Email: jason.shapiro@mountsinai.org
Appl Clin Inform 2015; 6: 185-199

http://dx.doi.org/10.4338/ACI-2014-10-RA-0090

received: October 15, 2014

accepted: February 11, 2015

published: March 25, 2015

Citation: Garg N, Husk G, Nguyen T, Onyile A, Echezona S, Kuperman G, Shapiro JS. Hospital closure and insights into patient dispersion: the closure of Saint Vincent's Catholic Medical Center in New York City. Appl Clin Inf 2015; 6: 185-199

http://dx.doi.org/10.4338/ACI-2014-10-RA-0090 


\section{Background}

Hospital closures have significant impact on their surrounding communities [1-4]. In the United States, over 300 hospitals have closed in the last 20 years, and in urban areas, about ten hospitals have closed per year for the last five years [3]. Hospitals close for a variety of reasons, generally centered on financial debt, mergers, and inability to make a profit [5-7]. Hospital closures have been associated with worsened healthcare for the community, especially for the most vulnerable populations [2, 8-10]. For example, closures have been associated with increased rates of readmission and increased distances to the nearest healthcare facility, which can impact the outcomes of trauma patients and those with heart attacks $[1,2,8]$. Remaining hospitals in the area divert ambulances to other hospitals at an increased rate and are increasingly crowded as a result of hospital closures [9, $11]$.

Knowing what patients do in the setting of a hospital closure is essential to appropriately plan for patient care at remaining provider organizations in the area. For example, in an emergency department, an unplanned influx of patients beyond expectation may lead to crowding which adversely affects patient outcomes [11, 12]. Furthermore, crowding may affect more than just the care at the hospital. Ambulances that would have gone to the closed hospital are rerouted to the next nearest hospital until these hospitals become crowded and then patients must travel even farther to receive healthcare $[1,9]$. In addition, other nearby hospitals could run the risk of not having the capacity to safely manage the increased census created by the shut-down facility, especially if the increase is sudden and extreme [13].

Studies show that patients use a number of factors to choose where to seek care, including proximity, insurance, general reputation, the individual's healthcare needs, wait times, and loyalty to a particular hospital or provider [14-16]. These factors may weigh differently for various services. For example, unlike other parts of a hospital, emergency care is usually unplanned. In such scenarios, loyalty to a provider may be less of a contributing factor in a patient's decision compared to wait times or how quickly one can reach the hospital. Patients transported by ambulance may be brought to the nearest 911-receiving hospital, independent of their hospital choice. On the other hand, ambulatory care is usually planned and thus patients may worry more about insurance and reputation of the provider over proximity and wait times.

In light of these differences, the location where patients seek care in response to a hospital closure may be different for each encounter type. To our knowledge, there is no comprehensive analysis in the current literature of where patients go after a hospital closes.

\section{Objectives}

In this paper, we aim to describe the impact of the 2010 closure of Saint Vincent's Medical Center (SVCMC), a medical center in lower Manhattan in New York City, on the number of emergency, inpatient, and ambulatory patient encounters to eight surrounding hospitals. Furthermore, we analyze the correlation of the size of the dispersion of SVCMC patients to each of the other hospitals with distance of each from SVCMC.

\section{Methods}

\subsection{Setting}

Founded in 1849, Saint Vincent's Catholic Medical Center (SVCMC) became a pillar of the Manhattan healthcare community seeing over half a million patients per year by the 2000s [17]. It also provided medical attention to the victims of several historic disasters, including the cholera epidemic of 1849, the sinking of the Titanic in 1912, the 9/11 attack in 2001, and the Hudson River landing of US Airways Flight 1549 in 2009 [18, 19]. In the 2000s, SVCMC developed increasing financial debt and despite funding from the government, was forced to cease operations. On April $6^{\text {th }} 2010$, SVCMC announced that it would be closing its doors to patients by the end of the month [20]. On April 16 ${ }^{\text {th }}$, 
ambulances stopped bringing patients to SVCMC. On April $30^{\text {th }}$, SVCMC ceased providing all emergency and inpatient services $[17,18]$. Some ambulatory services continued until May 31, 2010 when these practices were transferred to other hospitals [21].

The New York Clinical Information Exchange (NYCLIX) was a not-for-profit health information exchange (HIE) that began in early 2009 and was functioning during the time of the SVCMC closure. An HIE allows for the exchange of health data electronically across different sites and different electronic health record systems. In addition, a single patient can be linked to several sites and systems simultaneously and healthcare utilization information can be collected [22]. NYCLIX provided an HIE capability for the majority of hospitals and hospital patients in Manhattan from 2009 to 2011 before it merged with another HIE [23]. NYCLIX data provided a way to understand where patients who had been receiving care at SVCMC sought care after SVCMC's closure.

The analysis includes data from NYCLIX for eight other hospitals, seven of which are also in the borough of Manhattan. The hospital not in Manhattan is located in a neighboring borough and actually closer to SVCMC than some of the 12 total Manhattan hospitals. The specific hospitals are not identified by name in this study in compliance with NYCLIX policy and are referred to as Sites A-H, in order of increasing distance from SVCMC. All hospitals are privately owned, not-for-profit, academic hospitals. In the year 2006, these eight hospitals comprised 7,242 inpatient beds, 538,510 ED visits, and 349,217 hospital discharges. The seven hospitals in Manhattan accounted for 76.6\% of Manhattan ED visits [24].

\subsection{Framework for Analysis}

Specific queries were run by a NYCLIX staff member acting as honest broker, which is a neutral intermediary between the person whose data is being studied and the researcher. The honest broker works with identifiable data as part of his operational duties, and was able to create a coded, deidentified aggregate level research data set for our analysis [25]. Data included daily counts of SVCMC patients visiting the other sites in NYCLIX, along with the type of encounter (ED, inpatient or ambulatory). SVCMC patients were defined as any patient with at least one prior encounter, of any type, to SVCMC since NYCLIX was implemented in March 2009. Three distinct time periods were chosen for analysis:

- Pre-closure: January 5, 2010 - April 5, 2010

- Peri-closure: April 6, 2010 - April 30, 2010

- Post-closure: May 1, 2010 - July 30, 2010

The pre-closure time period was defined as the thirteen weeks prior to the first announcement in the press that the hospital would be closing, which occurred on April 6, 2010. The peri-closure time period was defined as the period from that first announcement until the date of actual closure, April 30, 2010. Post-closure was defined as the thirteen weeks following the closure date. The peri-closure period was chosen as a separate time period for the analysis because after announcement of closure it was assumed that public knowledge of the imminent closure would bias patient's choice of hospital in an unpredictable manner. The research protocol was reviewed by the Beth Israel Institutional Review Board, which determined the study was exempt.

\subsection{Analytic Approach}

\section{Differences between pre- and post-closure periods}

We first measured the average number of SVCMC patient encounters in the pre- and post-closure periods by encounter type for each hospital and for the eight NYCLIX sites collectively. Since the number of SVCMC patients was increasing over time, we calculated the rate of encounters of SVCMC patients at other sites on any given day "per 100,000 SVCMC patients." The differences between those averages were calculated per type of encounter per site and single-factor ANOVA was used to calculate a p-value for each of these differences ( $>$ Table 1$)$. 


\section{Correlation between distance from SVCMC and number of encounters}

Next, we measured driving distance and travel time in minutes using public transportation between SVCMC and other sites. Distances in miles correlates well with driving distance in New York City using Google Maps and also gave an absolute number of miles. These distances in miles and public transportation travel times from SVCMC were obtained from Google Maps and the lowest number of miles resulted under the "Driving/Taxi" tab and the lowest number of minutes resulted under the "Public Transit" tab were recorded. We then correlated the travel distance for each site to the change in SVCMC patient encounters from pre- to post-closure for ED, inpatient, and ambulatory encounter types ( $>$ Figure 1). A chi-square analysis was applied to determine if the closest hospital had a strong correlation with significant increases in SVCMC patients. We also looked for specific distances, beyond which we could expect to see a change in the behavior of SVCMC patients.

\section{Graphical representation of encounters over time}

Finally, the total number of SVCMC patient encounters per day was graphed for each hospital by emergency department, inpatient, and ambulatory encounter types to give a visual representation of the changes over time. SVCMC patient encounters were displayed graphically using a 14-day centered moving average to convey trends in patient encounters. The NYCLIX total was graphed for all three encounter types ( $>$ Figure 2 ), and then 14-day moving averages for each site were plotted on a separate graph for each encounter type ( $>$ Figures 3-5).

\section{Results}

The total number of SVCMC patients at the beginning of the study period was 114,121 and it increased to 130,423 by the end (a $14.3 \%$ increase). Overall, the rate at which the number of SVCMC patients increased per day was 156.6 during the pre-closure period, which is the number of patients per day who were new to SVCMC since March 2009 when NYCLIX began exchanging data with all sites. During the peri-closure period, there were about 120.9 new SVCMC patients per day from April 6 - April 15 and then 45.3 from April 16 - April 30. This decrease of 37.5\% correlates well with when ambulance services to SVCMC were terminated (April 15, 2010).

Four of the eight hospitals saw statistically significant changes in the number of SVCMC patient encounters per 100,000 SVCMC patients in their ED, inpatient, and / or ambulatory encounters from pre-closure to post-closure. These sites were A, B, E, and G ( $\triangleright$ Table 1). For ED encounters, Site A and Site B had significant increases of 7.7 and 1.17 encounters per 100,000 SVCMC patients per day, respectively. For inpatient encounters, Site A had an increase of 4.18 encounters per 100,000 SVCMC patients per day and Site G had a small but significant decrease of 0.39 . More sites had significant increases in ambulatory encounters than ED or inpatient encounters with Sites E, G, and A increasing 29.34, 19.98 and 3.71 encounters per day per 100,000 SVCMC patients, respectively. When combined, all hospitals saw a significant increase in all three types of encounters as seen by the row labeled "NYCLIX."

For the ED, the two sites with significant increases in the number of SVCMC patient encounters were the next two nearest hospitals, with the closest one, Site A at 1.3 miles, having an increase 6.58 times greater than the next closest one, Site B, at 1.9 miles from SVCMC, $7.70(\mathrm{p}<0.001)$ versus 1.17 $(\mathrm{p}=0.0005)(>$ Table 1$)$. Site A also had the shortest public transit time from SVCMC at 12 minutes. Hospitals outside a two mile radius of SVCMC had no significant increase in the number of ED encounters by SVCMC patients after SVCMC's closure.

The closest hospital - Site A - was the only hospital to have a significant increase in inpatient admissions ( Table 1). Site G, one of the farthest hospitals from our list of eight hospitals, had a small but significant decrease in the number of SVCMC inpatient patient admissions post-closure by 0.39 patients per 100,000 SVCMC patients per day $(\mathrm{p}<0.05)$. Finally, ambulatory sites saw no correlation of increases in SVCMC patient encounters with decreased distances from SVCMC ( $>$ Table 1). In fact, Site $E$ had the largest significant increase in the number of SVCMC ambulatory patient encounters among three sites and yet was the farthest away $(\mathrm{p}<0.05)$. There was no correlation with public transit times. 
When differences in pre- and post-closure were graphed per encounter type against distance from SVCMC, there was a significant correlation with the next closest hospital $(\mathrm{p}<0.005)$. When broken down, each encounter type had varying degrees of correlation with the next closest hospital with inpatient having the strongest and ambulatory having the weakest. With respect to specific distances, hospitals within a 2 mile radius could expect to see a significant increase in SVCMC patients with a p-value of less than 0.01 .

The number of SVCMC patient encounters at other sites after SVCMC closed increased for all encounter types ( Figure 1). The combined ED encounters showed a dramatic increase in SVCMC patients immediately after announcement of closure ( Figure 2). Inpatient encounters by SVCMC patients mirrored ED, but with a lower magnitude ( Figure 2). Ambulatory encounters at other sites also increased slightly after closure but a more dramatic increase was seen after May $31^{\text {st }}(\nabla$ Figure 2), which is the date that SVCMC Ambulatory sites stopped seeing patients. Figure 3 and - Figure 4 show a significant increase in both ED and inpatient encounters for Site A, but other sites have no visually discernible increase. A gradual increase in ambulatory encounters is seen at the time of SVCMC's closure for Site A, and then a more abrupt, large but delayed increase is seen for Site E ( $>$ Figure 5).

\section{Discussion}

In this study, the NYCLIX dataset was used to analyze the impact of a closing hospital on ED, inpatient, and ambulatory encounters at nearby hospitals. Among the hospitals in the dataset, the next closest hospital to SVCMC by both absolute distance in miles and by travel time appears to bear the greatest burden of ED and inpatient encounters from SVCMC patients. The distances to the next closest hospitals were between 1-2 miles. While our study was of a single closing hospital in an urban setting, it is likely that patients of a closing hospital in any setting go to the next closest hospital for their emergency and inpatient medical needs, though this needs further investigation. This information could help inform the allocation of resources during future hospital closures, as preparation for increased patient influx may help mitigate crowding and improve patient outcomes at the nearest hospitals.

Interestingly, Site B saw a greater increase in ED encounters than Site C even though the public transit time for Site C was slightly shorter. Site B is closer than Site C by 1.2 miles, indicating that absolute distance may matter more than public transit time for ED encounters. One explanation for this may be that a substantial proportion of patients were transported by ambulance, private car or taxi. Another explanation may be that closer absolute distance may correlate with familiarity with the hospital and / or the general neighborhood, which could factor in to a patient's decision making.

Ambulatory encounter changes at other sites in the NYCLIX database after SVCMC closure behave categorically differently from ED and inpatient encounter changes. Ambulatory encounters do not appear to correlate with distances at all, and the increases at other sites happened well after the closure date of SVCMC. The increase in ambulatory encounters of SVCMC patients occurred roughly two months after the first announcement of SVCMC closure, which correlates with when SVCMC closed the remaining of their ambulatory services (May $31^{\text {st }}$ ). Among the hospitals in the NYCLIX database, Site E had the greatest increase in the number of SVCMC patient encounters but was farthest away among those with significant increases in ambulatory encounters. Part of the reason for this may be the physicians with ambulatory practices associated with SVCMC became part of another hospital system and retained their patient population. By deduction from the data in this analysis, the three most probable places the SVCMC staff relocated are Sites E, A, and G in order of decreasing magnitude. Site A actually saw an earlier increase, which may be attributable to the fact that it was the closest, and that some SVCMC physicians may have also had privileges at Site A, as is often the case for hospitals in close proximity, whereas other SVCMC physicians had to wait for privileges at hospitals farther away. This effect of credentialing delays may also be seen on the inpatient side with respect to elective admissions for surgeries and admissions from ambulatory practices.

One important limitation of the study is that although the majority of the hospitals closest to SVCMC participated in NYCLIX, many of the approximately 80 hospitals in the 5 boroughs of New 
York City and the surrounding New York counties did not, and some unknown number of SVCMC patients, as defined in this study, likely sought care at these other hospitals [24]. Notably absent from this study are the 11 public hospitals in the New York City Health and Hospitals Corporation that serve 1.4 million patients every year, including Bellevue Hospital Center. Adalja et al published a study that included survey data from the four closest hospitals to SVCMC on increases in ED patient census after one month of closure. They also trended Bellevue ED census over time for the year of 2010 and compared that to ED census of prior years. Their data was not exclusive to SVCMC patients though, and they also only assessed ED volume. It is not known how much of an effect missing data from hospitals not included in the study [25]. It is not known how much of an effect missing data from hospitals not included in this study may have had on our results, though there were only two hospitals within a two mile radius of SVCMC not included in our study. Despite the limitation of having incomplete coverage of Manhattan, the described methods for using a health information exchange for this type of analysis is still of great value.

Another limitation of this study is that it only captures patients who had at least one recorded encounter to SVCMC between March of 2009, when NYCLIX began exchanging data with all sites, and prior to being seen at one of the other sites during the study period. Thus, there were likely many patients with at least one SVCMC encounter prior to March of 2009 who were not captured in the NYCLIX dataset. Pre-closure, the total number of SVCMC patients in NYCLIX was increasing by about 156.6 per day, indicating that the NYCLIX dataset of SVCMC patients had not yet reached a steady state or leveled-off by the time of the SVCMC closure. Further illustrating this limitation, NYCLIX data accounts for only a 3.1\% increase in the Site A ED census during the study period, a fraction of the overall increase actually seen following the SVCMC closure [26].

Other important limitations to discuss are that patients who have had just one prior SVCMC visit may not have been true "SVCMC patients." There is probably some effect captured from people who just went to SVCMC once but were normally a patient at another facility. The absolute totals of SVCMC patients seen at the nearest hospitals may be biased in this regard. Alternate definitions that look at the pattern of visits in a more complex way may be a better reflection of the true number of "SVCMC patients," but we chose a more sensitive definition in order to identify as many potential patients as possible. Similarly, patients who were seen multiple times in the post-closure period, including for re-hospitalizations, frequent ED users, or for follow up visits within the three month period were not excluded after their first visit to one of the other sites, possibly inflating post-closure numbers. Our data also did not permit us to control for seasonality of patient illnesses, which could have also affected the differences seen in the number of SVCMC patient encounters between the pre-closure period and post-closure period at one site. In the instances where seasonality of diseases is higher prior to April, differences in pre- and post-closure encounters at a site may have been missed. In the instances where seasonality of diseases is higher after April, differences in pre- and post-closure may be skewed to be greater than they actually were.

In this study, using data obtainable from a health information exchange, we determined that patients who received care at a closing hospital may have been most likely to visit the closest available hospital for their emergency and inpatient medical care. This trend did not extend to ambulatory services, where the actual distance from the closing hospital to other sites did not appear to correlate with SVCMC patient encounters. It would be helpful to see if similar results could be found in other regions where hospital closures have occurred. Future studies should also focus on generating a model to predict the timing and magnitude of an increase in number of patients seen at the next nearest hospital of a closing hospital so that hospitals can prepare for sudden surges in their patient volume.

\section{Conclusion}

To our knowledge, this is the first analysis of an HIE to investigate the behavior of patients who received care at a closing hospital. Without an HIE, this type of analysis would be difficult or impossible. Although a similar analysis could theoretically be performed using claims data, the payer market in New York City is highly fragmented, and there is a large number of uninsured patients, making such a study infeasible. A data set with more hospitals and a longer timeframe could improve the 
validity of results. There are also more clinically interesting questions that could be asked if more detailed data were available, such as which types of patients may not be getting access to care and what the impact on clinical outcomes is when a hospital closes.

In 2013, there were 315 data exchange initiatives identified in the nation at various stages of implementation and efficacy [27]. With improvements and expansion of HIE and other forms of interoperable health information, it will become increasingly possible to conduct studies that track patients across sites, and gain a better understanding of how patients actually access care. In the case of hospital closures, HIEs across the United States may provide useful data for predictive modeling and advanced preparation for the remaining hospitals in a region. Additional studies using HIE data may also help inform policy makers regarding the decision to close hospitals in various regions.

\section{Clinical Relevance Statement}

Hospital closures are increasingly common, and healthcare delivery for unplanned hospital closures can lead to crowding in other healthcare facilities and poor patient outcomes. Through advances in health information exchange, we can learn from hospital closures in the past to help plan for ones in the future. According to the example in this research, patients from a closing hospital will go to the next nearest hospital for their emergency and inpatient needs, and the increase in patient visits from a closing hospital will happen prior to the final closure date of the closing hospital.

\section{Conflicts of Interest}

The authors declare that they have no conflicts of interest in the research.

\section{Protection of Human and Animal Subjects}

The research protocol was reviewed by the Beth Israel Institutional Review Board, which determined the study was exempt.

\section{Acknowledgements}

There are no formal acknowledgements for this research. 


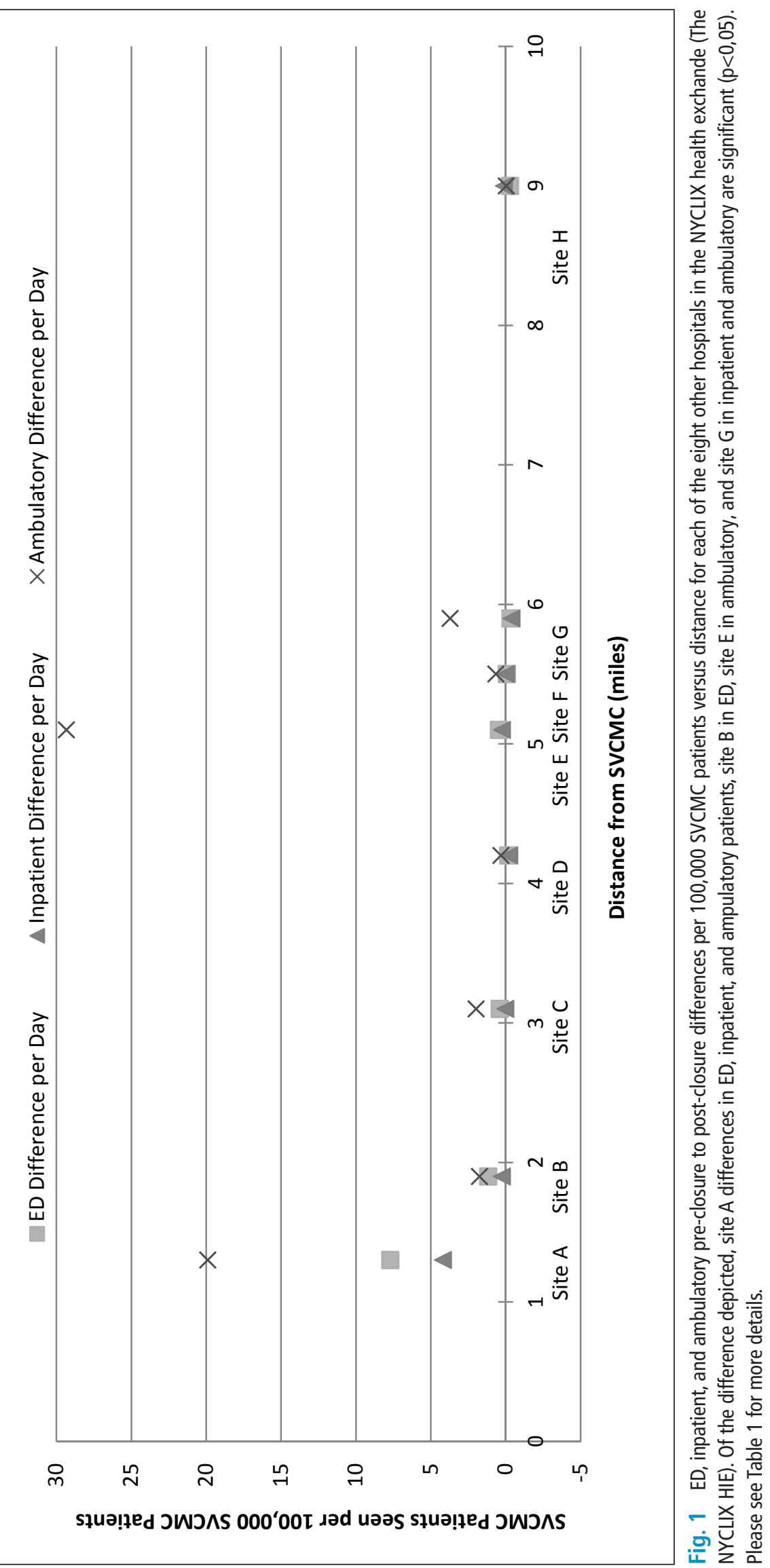




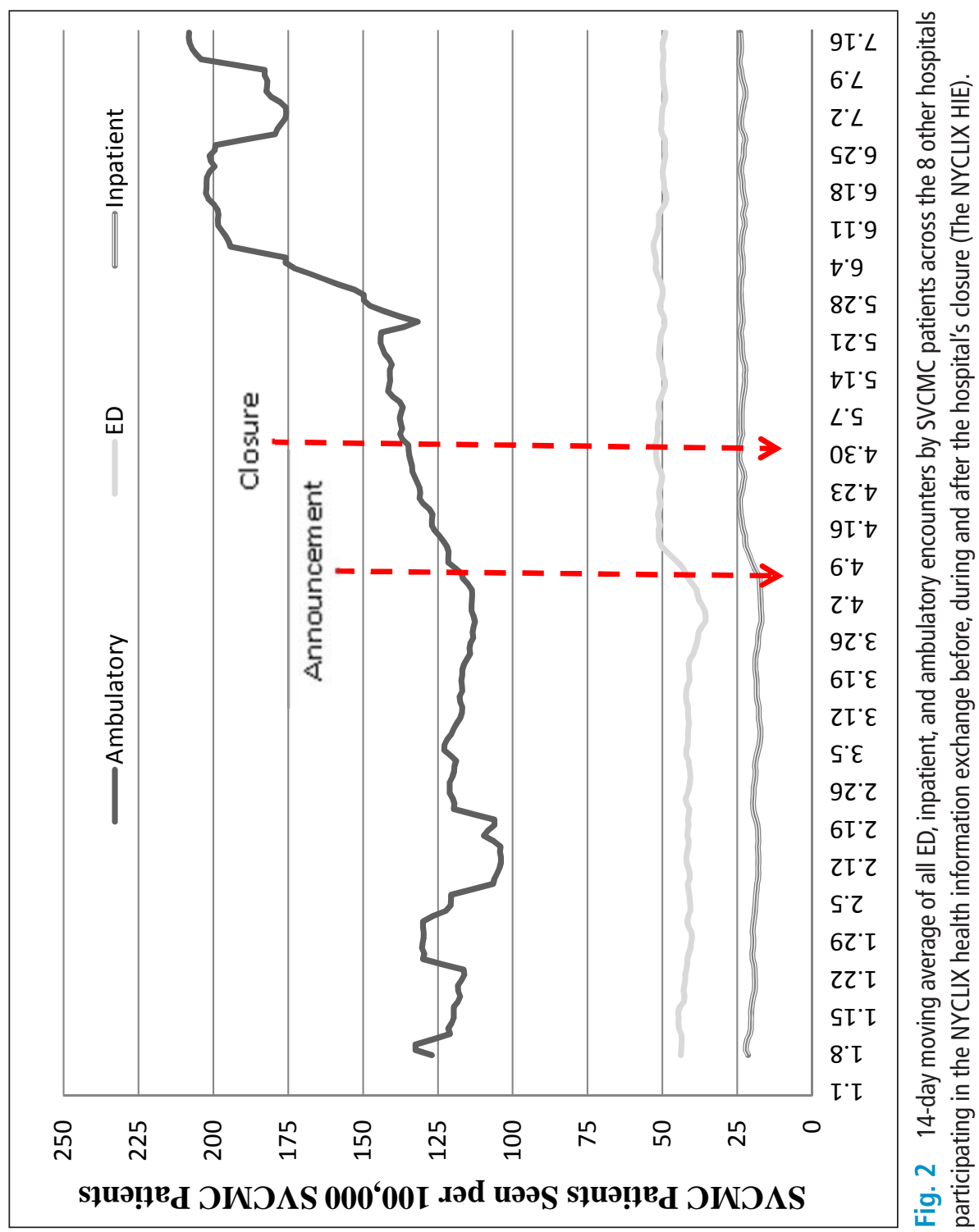




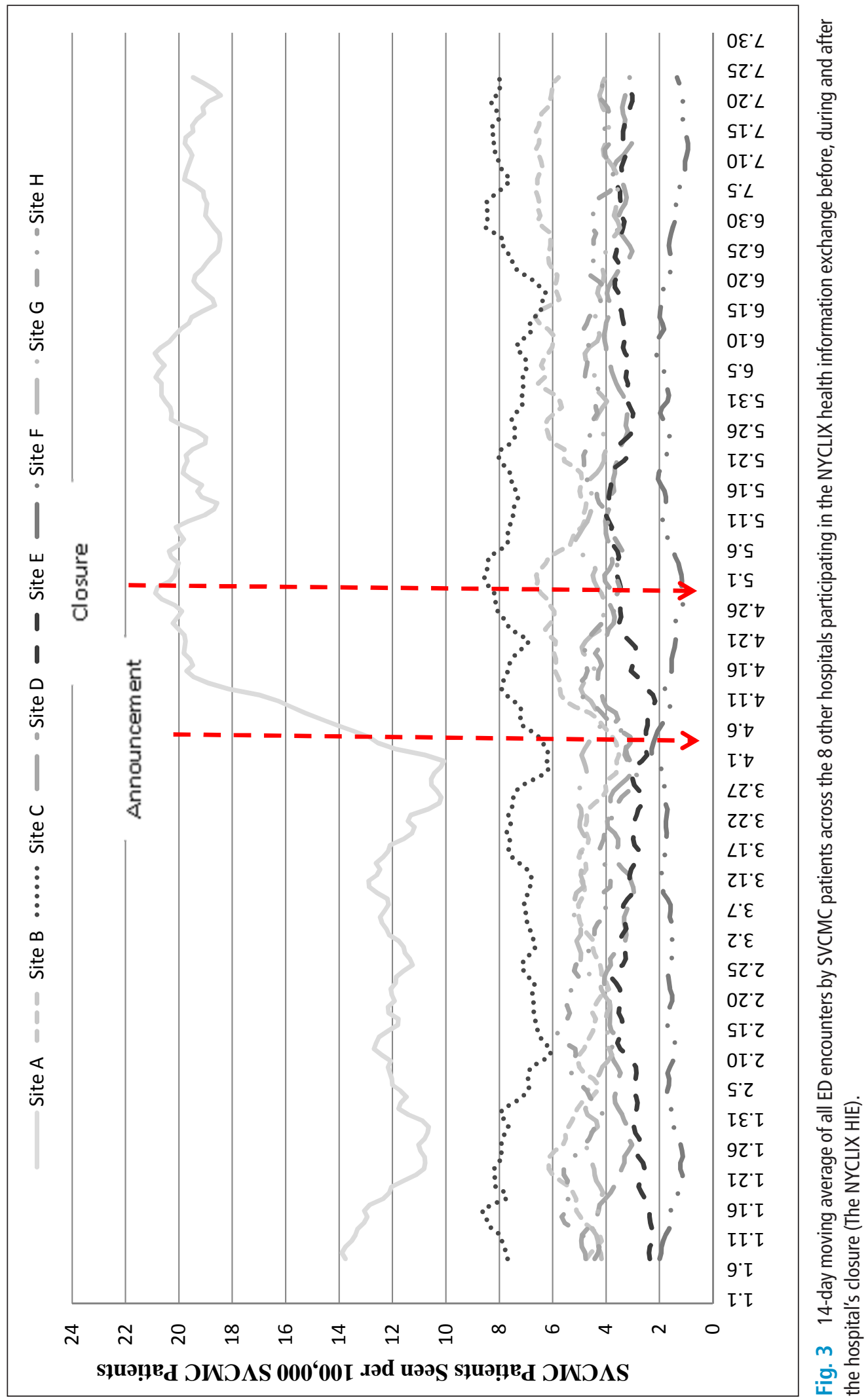




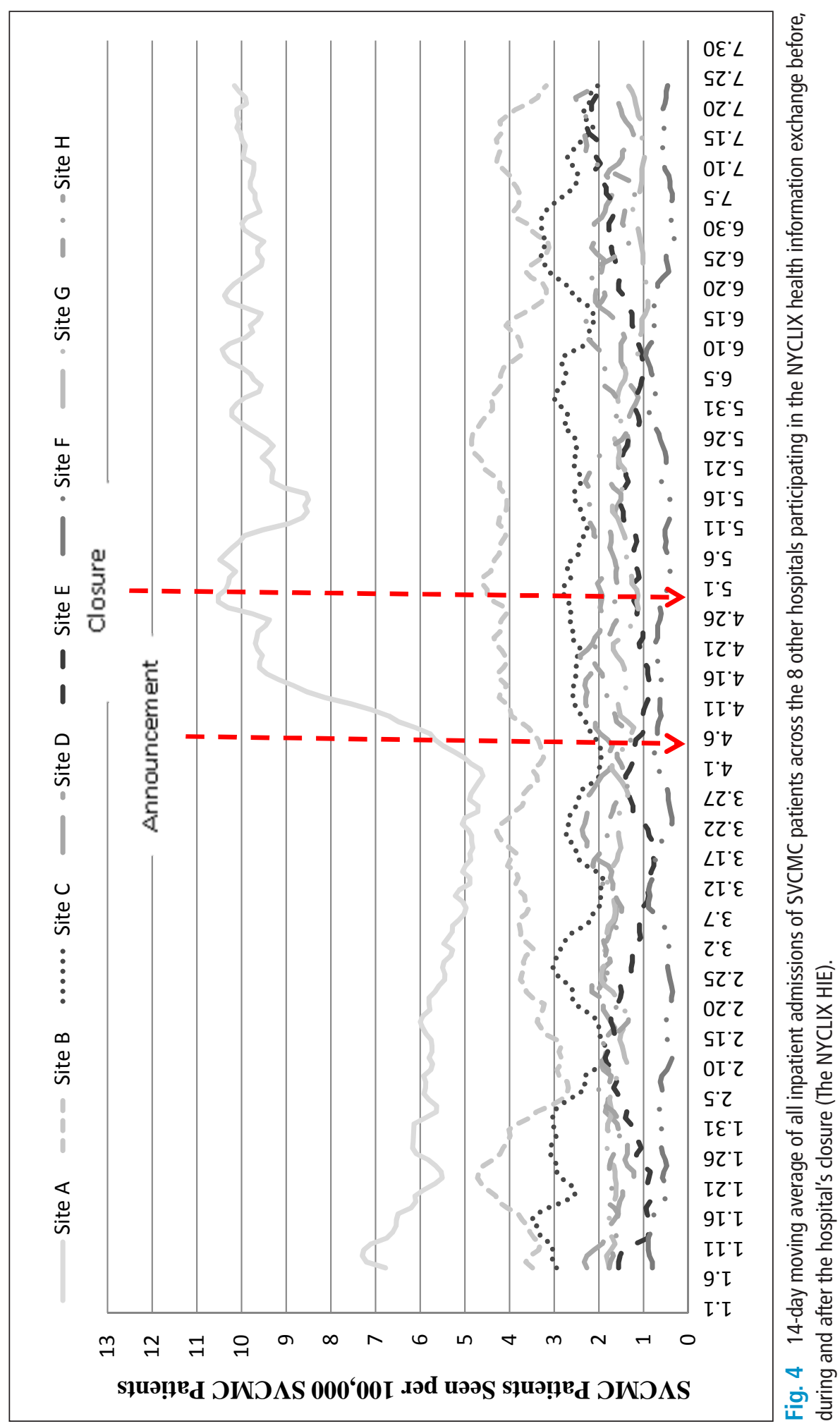




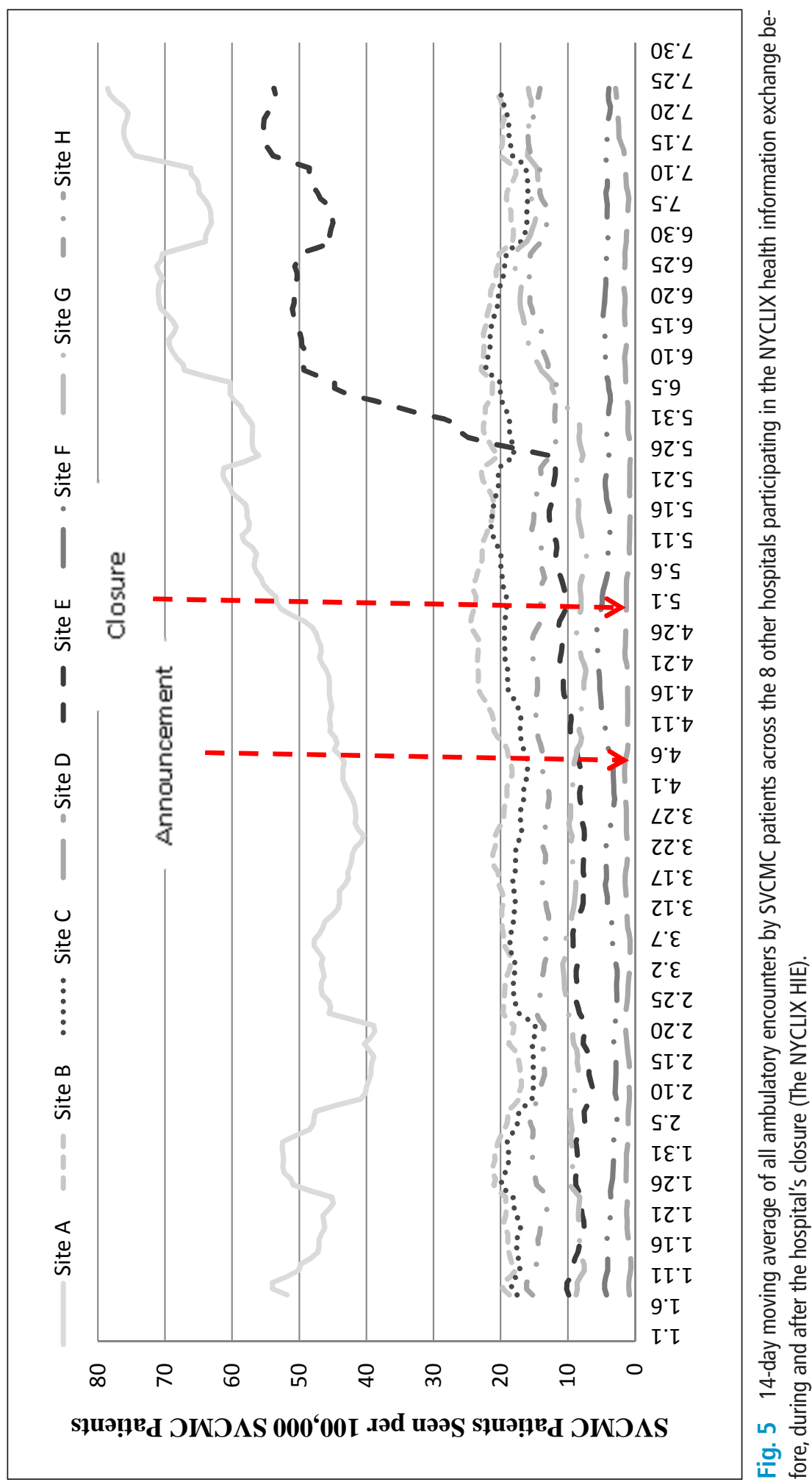


Table 1 Distance and travel times from SVCMC to each site, with the difference in ED, inpatient, and ambulatory encounters per 100,000 SVCMC patients in the NYCLIX database pre- and post-closure of SVCMC. Numbers in bold denote a p-value of $<0.05$ using ANOVA.

\begin{tabular}{|l|c|c|c|c|c|}
\hline Sites & $\begin{array}{l}\text { Distance } \\
\text { (mi) }\end{array}$ & $\begin{array}{l}\text { Public } \\
\text { Transit } \\
\text { (min) }\end{array}$ & $\begin{array}{l}\text { ED Difference } \\
\text { (encounters/ } \\
\text { day) }\end{array}$ & $\begin{array}{l}\text { Inpatient } \\
\text { Difference } \\
\text { (admissions/day) }\end{array}$ & $\begin{array}{l}\text { Ambulatory } \\
\text { Difference } \\
\text { (encounters/day) }\end{array}$ \\
\hline Site A & 1.3 & 12 & 7.70 & 4.19 & 19.90 \\
\hline Site B & 1.9 & 25 & 1.17 & 0.25 & 1.76 \\
\hline Site C & 3.1 & 20 & 0.39 & 0.01 & 1.97 \\
\hline Site D & 4.2 & 32 & -0.18 & -0.24 & 0.31 \\
\hline Site E & 5.1 & 30 & 0.45 & 0.25 & 29.34 \\
\hline Site F & 5.5 & 32 & -0.08 & -0.03 & 0.66 \\
\hline Site G & 5.9 & 34 & -0.32 & -0.39 & 3.71 \\
\hline Site H & 9 & 33 & -0.29 & 0.12 & -0.04 \\
\hline NYCLIX & & & 8.80 & 4.14 & 56.38 \\
\hline
\end{tabular}




\section{References}

1. Buchmueller TC, Jacobson M, Wold C. How far to the hospital?: The effect of hospital closures on access to care. J Health Econ [Internet] 2006;25(4):740-61. Available from: http://www.sciencedirect.com/science/ article/pii/S0167629605001116

2. Rahman M, Zinn JS, Mor V. The Impact of Hospital-Based Skilled Nursing Facility Closures on Rehospitalizations. Health Serv Res 2012;:n/a-n/a. Available from: http://dx.doi.org/10.1111/1475-6773.12001

3. Number of Hospitals (Table 2.1). 2013.

4. McLafferty S. Neighborhood characteristics and hospital closures. A comparison of the public, private and voluntary hospital systems. Soc Sci Med 1982; 16: 1667-1674.

5. Longo DR, Chase GA. Structural determinants of hospital closure. Med Care 1984; 22: 388-402.

6. Dafny LP. Hospital Industry Consolidation - Still More to Come? N Engl J Med 2014; 370(3): 198-199.

7. Harrison TD. Consolidations and closures: An empirical analysis of exits from the hospital industry. Health Econ 2007; 16: 457-474.

8. Hsia RY-J, Shen Y-C. Rising Closures Of Hospital Trauma Centers Disproportionately Burden Vulnerable Populations. Health Aff 2011; 30: 1912-1920.

9. Sun BC, Mohanty SA, Weiss R, Tadeo R, Hasbrouck M, Koenig W, Meyer C, Asch S. Effects of Hospital Closures and Hospital Characteristics on Emergency Department Ambulance Diversion, Los Angeles County, 1998 to 2004. Ann Emerg Med 2006; 47(4): 309-316. Available from: http://www.sciencedirect. com/science/article/pii/S0196064405019918

10. Bindman AB, Keane D, Lurie N. A public hospital closes. Impact on patients' access to care and health status. JAMA 1990; 264: 2899-2904.

11.Zemla R. City Hospitals Face Overcrowding [Internet]. Columbia Spect. 2008;Available from: http://www. columbiaspectator.com/2008/02/29/city-hospitals-face-overcrowding

12.Bernstein SL, Aronsky D, Duseja R, Epstein S, Handel D, Hwang U, McCarthy M, John McConnell K, Pines JM, Rathlev N, Schafermeyer R, Zwemer F, Schull M, Asplin BR.. The Effect of Emergency Department Crowding on Clinically Oriented Outcomes. Acad Emerg Med 2009; 16(1): 1-10. Available from: http://dx.doi.org/10.1111/j.1553-2712.2008.00295.x

13. Guttmann A, Schull MJ, Vermeulen MJ, Stukel TA. Association between waiting times and short term mortality and hospital admission after departure from emergency department: population based cohort study from Ontario, Canada. BMJ 2011; 342.

14.Blizzard RDBA. Healthcare Panel: How do People Choose Hospitals? Gallup 2005; Available from: http://www.gallup.com/poll/19402/healthcare-panel-how-people-choose-hospitals.aspx

15.De Groot IB, Otten W, Dijs-Elsinga J, Smeets HJ, Kievit J, Marang-van de Mheen PJ. Choosing between Hospitals: The Influence of the Experiences of Other Patients. Med Decis Mak 2012;

16. Lee WI, Shih BY, Chung YS. The exploration of consumers' behavior in choosing hospital by the application of neural network. Expert Syst Appl 2008; 34: 806-816.

17. NewellNYC. Timeline of Events | March - April 2010 [Internet]. 2010;(November 3, 2012). Available from: http://www.slideshare.net/NewellNYC/st-vincents-timeline

18. Mokha K and SG. St. Vincent's Hospital closes for good after 160 years; treated victims from Titanic to 9/11 [Internet]. NY Dly. News. 2010;Available from: http://articles.nydailynews.com/ 2010-04-30/local/27063193_1_lab-worker-end-inpatient-services-24-hour-urgent-care-center

19. Saint Vincent's Catholic Medical Center. 2012;

20.St. Vincent's Hospital Manhattan [Internet]. New York Times. 2010;Available from: http://topics.nytimes.com/top/reference/timestopics/organizations/s/st_vincents_hospital_manhattan/index.html

21.Benson Gale Scott, and Irina Ivanova B. The Closure Plan. Crain's Heal. Pulse. 2010;2013(September 17, 2013):Business article on plan to continue to hold SVCMC.

22. Moore T Doles L, Calman N, Camhi E, Check T, Onyile A, Kuperman G. SJS. A clinical notification service on a health information exchange platform. 2012;

23. Shapiro JS, Kannry J, Kushniruk AW, Kuperman G, New York Clinical Information Exchange Clinical Advisory S. Emergency physicians' perceptions of health information exchange. J Am Med Inf Assoc 2007; 14(6): 700-705.

24.New York State Department of Health. Statewide Planning and Research Cooperative System (SPARCS) [home page on the Internet]. Albany (NY): 2013. Available from: http://www.health.ny.gov/statistics/ sparcs/

25.FAQs, Terms and Recommendations on Informed Consent and Research Use of Biospecimens: The Secretary's Advisory Committee on Human Research Protections (SACHRP) [Internet]. Washington, D.C.: 2011. Available from: http://www.hhs.gov/ohrp/sachrp/commsec/attachmentdfaq'stermsandrecommen dations.pdf.pdf 
26. Adalja Matthew Watson, Samuel Wollner, Kunal J. Rambhia, and Eric S. Toner AA. Response to the Sudden Closure of St. Vincent's Hospital: Learning from a Real, No-notice, Prolonged Surge Event. Biosecurity Bioterrorism Biodefense Strateg Pract Sci 2011; 9(2): 153-161.

27. Results from Survey on Health Data Exchange 2013. Washington, D.C.: 2013. 